\title{
Computational Approaches to Understanding the Self- Assembly of Peptide-Based Nanostructures
}

\author{
Tell Tuttle*[a]
}

\begin{abstract}
The interest in the self-assembly of peptide-based systems has grown significantly over the past $10-15$ years as more and more applications are shown to benefit from the useful properties of the amino acid based monomers. With the desire to apply the principals of self-assembly to systems within new application areas there has been an increasing emphasis in understanding the governing forces involved in the self-assembly process and using this understanding to predict the behavior of, and design, new materials.
\end{abstract}

Keywords: peptide $\cdot$ self-assembly $\cdot$ computational $\cdot$ DFT $\cdot$ coarse grain

\section{Introduction}

Self-assembly is a process by which monomers (discrete units) spontaneously interact with each other to form ordered structures. There are a large number of different chemical systems that are known to undergo self-assembly. However, peptide-based systems are of particular interest due to their biocompatibility and their ability to form varied supramolecular structures with only small variations in the individual monomers. ${ }^{[1]}$

The amino acids that compose a peptide have particular properties that govern their intermolecular interactions. Depending on the nature of the side chain, the amino acids are able to interact, to varying degrees, via ionic and electrostatic interactions, H-bonding interactions, and dispersion-based interactions. While the initial aggregation of peptides is largely due to the hydrophobic effect, ${ }^{[2]}$ the ability to interact at close range to form ordered mesoscale systems critically depends on the specific interactions and as such, the primary sequence of the peptide. The ability of peptides to self-assemble was originally discovered for longer oligopeptides, ${ }^{[3]}$ however, the self-assembly of short peptides has resulted in the significant interest in this field over the past 15 years. ${ }^{[4]}$<smiles>N[C@@H](Cc1ccccc1)C(=O)N[C@@H](Cc1ccccc1)C(=O)O</smiles>

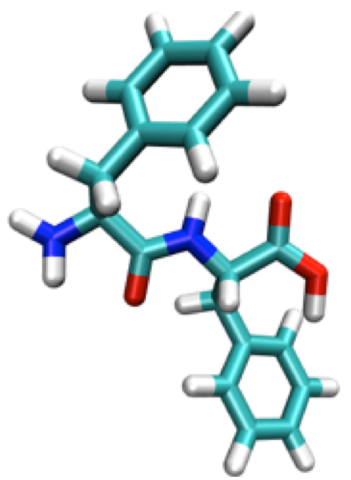

To this end, computational approaches have played an increasingly important role over the past decade in helping to decode how small changes in the primary structure can lead to significantly different nanostructures with new function. In this review a brief survey of the different computational approaches employed in this quest for understanding are provided, along with representative examples of the types of questions that can be answered with each of the different approaches.

Figure 1. Chemical structure and molecular model of diphenyalanine.

The first example of a dipeptide that was shown to self-assemble was diphenylalanine (FF, Figure 1). In 2001, Gorbitz ${ }^{[4 \mathrm{~b}]}$ used crystal structures to characterize the structure of diphenyalanine as nanotubes. Moreover, by comparing the structures formed by diphenylalanine and dileucine, Gorbitz was able to demonstrate that the different interactions between the sidechains, rather than just their hydrophobicity, were crucial for determining the resulting structure and as such, its potential applications. $^{\text {[4b, 5] }}$

In 2003, Gazit and Reches ${ }^{[4 a]}$ demonstrated the potential applications of self-assembling dipeptides in their paper that describes the use of FF to cast nanowires. The success of the FF dipeptide resulted in a several efforts to modify the core FF motif to derive new functionality and structure as slight modifications of the phenylalanine side chain can result in a change in the interactions the peptide can have. For example, the removal of the methyl group to convert diphenylalanine to diphenylglycine resulted in the formation of nanospheres rather than nanotubes. ${ }^{[6]}$ This loss caused the phenyl groups to become more rigid and unable to rotate so the overall packing of the peptides was

[a] Tell Tuttle

WestCHEM, Department of Pure and Applied Chemistry, University of Strathclyde 295 Cathedral Street, Glasgow, G1 1XL, UK phone: +441415482290 e-mail: tell.tuttle@strath.ac.uk

Supporting information for this article is available on the WWW under http://dx.doi.org/10.1002/ijch.201xxxxxx.((Please delete if not appropriate)) 
changed. Similarly, the addition of a third amino acid to the C-terminus resulted in a change in the types of structures that were produced. CFF, resulted in the formation of nanospheres, ${ }^{[7]}$ while FFF formed nanoplatelets. $^{[8]}$

Introducing a third amino acid into the peptide chain can affect the resulting structure through a number of different mechanisms. In the case of a dipeptide, the short chain length requires a close, well-ordered packing of the peptides to balance the electrostatic interactions between the $\mathrm{N}$ - and $\mathrm{C}$-termini. For FFF, the chain is slightly longer therefore an optimum pairing of the charged termini results in an elongation of the structure. In addition, the increased flexibility of the FFF chain promotes this sheet like structure to assemble. The change in shape is also thought to be due to the introduction of another $\mathrm{H}$-bonding interaction within the peptide. ${ }^{[9]}$ All three of these factors could conceivably contribute to the relative stability of the resulting nanostructure, however, it is difficult, if not impossible to determine, prima facie, which is the dominant effect.

There are a number of other factors that can also affect the self-assembling ability of peptides. The chirality of amino acids has also been shown to have a dramatic effect on the ability of a peptide sequence to self-assemble. ${ }^{[7 a, 10]}$ Marchesan et al, have shown that the natural tripeptide sequence, VFF, does not selfassemble. However, when the chirality of the amino acid at the $\mathrm{N}$-terminus was altered nanostructures were obtained, which led to the formation of a hydrogel. FTIR showed the amide region had shifted to lower frequencies suggesting that the presence of ordered $\beta$ sheets. These structural observations are not observed for the natural tripeptide. When the same process was carried out on FFV, where the $\mathrm{N}$-terminus amino acid was changed, similar observations were seen. There are also specific interactions that were observed through this process, where there is a shift in the peak indicating the presence of a deprotonated $\mathrm{C}$-terminus. This shift shows the presence of an interaction between the $\mathrm{COO}^{-}$ and the $\mathrm{NH}_{3}{ }^{+}$of the termini creating a strong electrostatic interaction. FTIR has not been the only way of observing the changes in structure. Visual inspection has shown that the sample with the D-amino acids at the N-terminus were able to hydrogelate, which was the first time this type of self assembly has been reported. The continuation of this work has shown the formation of structures with LFF. ${ }^{[10 d]}$ Similar to VFF and FFV, changing the chirality of the amino acid at the $\mathrm{N}$-terminus to the $\mathrm{D}$-amino acid induces the formation of structures and gelation.

Modifying the sequence length and the chirality of the peptides have both been shown to effect the selfassembly ability of a peptide. However, the most commonly used method for altering the self-assembly ability of short peptides is through modifying the termini of the peptide sequence. For example, by amidation of the C-terminus. This neutralizes the terminus and reduces charge-charge repulsions, which can prevent a structure from forming, although it also removes the potentially stabilizing head-to-tail arrangement whereby the positive $\mathrm{N}$ - and negative $\mathrm{C}$ termini are aligned. This is best seen in Cao et al., ${ }^{[11]}$ where groups are used to take away the charge of the termini reducing the electrostatic effect. In this study the authors used a series of sequential substitutions to rationalise the changes that were being observed in the resulting nanostructures. In particular, this study suggested that by substituting phenylalanine for tyrosine, the introduction of the hydroxide substituent makes the aromatic more electron deficient and inhibits the role of the $\pi-\pi$ stacking. The constraints on the structure are also relaxed as the $\pi-\pi$ stacking is not as predominant which allows for flexibility in the arrangement of the aromatic rings. The authors conclude that these reasons explain why FFK is able to form strong fibres, while FYK/YFK form weaker fibres and YYK does not form fibres.

While the small modification to the $\mathrm{C}$-terminus are successful in transforming some peptides sequences into self-assembling structures, the more common approach is to use aromatic moieties to functionalise the $\mathrm{N}$ terminus of the peptide. Aromatic peptide amphiphiles contain large aromatic groups, normally located at the $\mathrm{N}$-termini, which are used to induce self-assembly in peptide systems. ${ }^{[1]}$ There are many groups that can be used to induce the self-assembly of peptides, a selection of which are displayed in Figure 2.

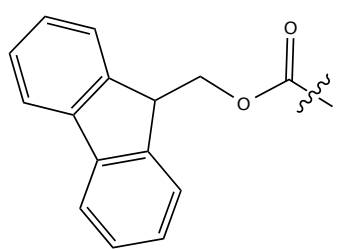

Fmoc

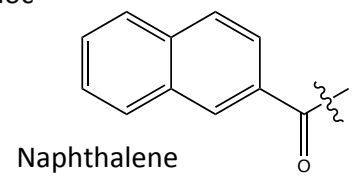

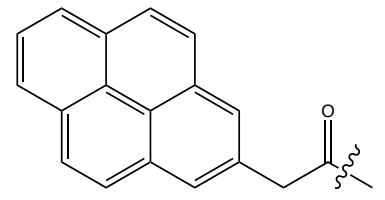

Pyrene

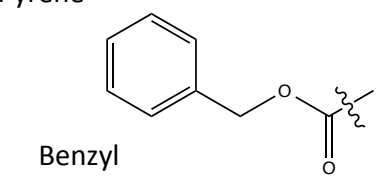

Figure 2. A selection of common aromatic motifs for inducing peptide self-assembly.

There are two main driving forces behind the formation of nanostructures from aromatic peptide amphiphiles. The first is the hydrophobic effect, which results from the fact that the water in a system will reorganize in such a way as to maximize their polar $(\mathrm{H}-$ bonding) interactions. The aromatic groups shown in Figure 2 present large surface areas with no favorable interactions for the water molecules to bind to and as such the hydrophobic molecules rearrange so that the least amount of water is in contact with the group. For planar molecules, the close packing of the rings ensures that the interactions of water with the hydrophobic rings are minimized. This is the hydrophobic effect. ${ }^{[12]}$ Although, it has been mentioned here that rings systems induce the hydrophobic effect, the same process happens for all hydrophobic groups, but with aromatic systems there is another interaction that takes over at short range and allows the formation of nanostructures, $\pi-\pi$ stacking.

The most predominant aromatic group that is used in peptide self-assembly is Fluorenylmethyloxycarbonyl, Fmoc. ${ }^{[13]}$ The main benefits for Fmoc are that the large 
ring system introduces a hydrophobic area to bring the molecules together and that the close-range $\pi$-stacking interactions are then able to create an ordered structure through self-assembly. ${ }^{[13 \mathrm{c}, 13 \mathrm{e}, 14]}$ The Fmoc moieties favor a parallel-displaced stacking interaction (Figure 3), which allows for the maximum stabilizing overlap between the $\pi$-system. This ability to promote peptides to self assemble allows for an increasingly diverse range of applications to be explored.

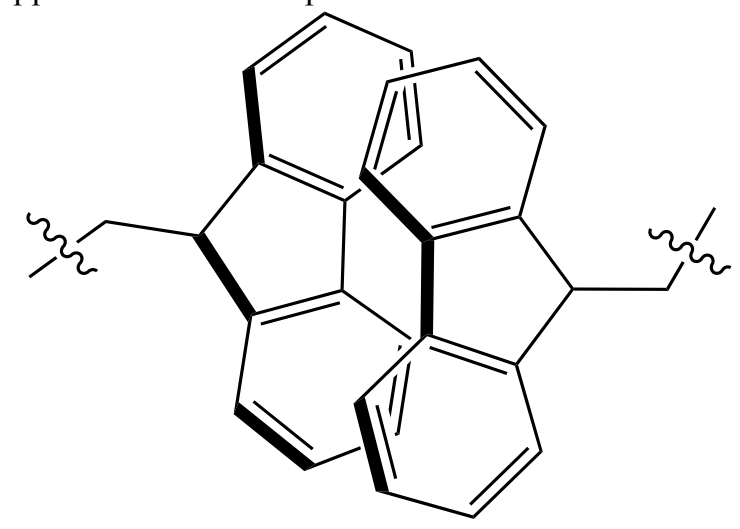

Figure 3. Arrangement of Fmoc $\pi$-stacking.

Ulijn and co-workers have been able to develop Fmoc functionalised peptides for use in cell culture. ${ }^{[13 \mathrm{c}]}$ Using Fmoc-FF as a self-assembled precursor, the subsequent addition of Fmoc-S creates a core shell structure where the hydrophobic Fmoc-FF fibres form a core, which is coated by Fmoc-S molecules with the polar serine residues facing towards the solvent. The core/shell structure provides greater flexibility in the design of the nanofibrous network and as such creates a more specialised environment for cells to grow.

The afore mentioned applications are a small sample of those currently being explored and already exploited by peptide-based self-assembly. However, the future development of new materials depends critically on understanding the types of non-covalent interactions (and their relative importance) that occur between the monomers and the organization of the self-assembled monomers of the resulting nanostructure at the atomic resolution. To this end, computational methods, within their model limitations, have played, and continue to play, an important role in driving forward our understanding of this class of materials.

Tell Tuttle's research is focused on the concept of "reducing molecular search spaces". The group works closely with experimental colleagues to address practical problems in the areas of molecular structure, molecular recognition, molecular reactivity and molecular properties. The understanding derived from the computational and theoretical methods employed in this research, results in the generation of predictive models that are used to design molecular-based solutions to a range of problems, from fields as diverse as fundamental chemistry through to drug discovery and from nanotechnology through to organic electronics.
The aim of this review is to provide an overview of the application of computational methods to study peptide self-assembly. Given this aim, the review does not provide a comprehensive study of the entire field, but instead considers the types of questions that each of the different computational methods may answer along with a typical case study from the literature to further illustrate the utility of the method. Within this review three computational methods (quantum chemistry, atomistic molecular mechanics, and coarse grained molecular mechanics) are described. The relative simplicity, and consequently computational efficiency, of the different methods results in their application across large size and time scales (Figure 4). Briefly, the most accurate and complex quantum chemistry methods are typically able to deal with 100's of atoms and only on the fs - ps timescale. Atomistic molecular mechanics methods are already significantly simplified (although still retain atomistic resolution) and can readily model $10,000-100,000$ atoms over the ns - $\mu$ s timescale. The loss of atomic resolution through grouping atoms into beads within coarse grained molecular mechanics results in a further simplification and the ability to readily model $100,000-1,000,000$ atoms over the $\mu \mathrm{s}-\mathrm{ms}$ timescale.

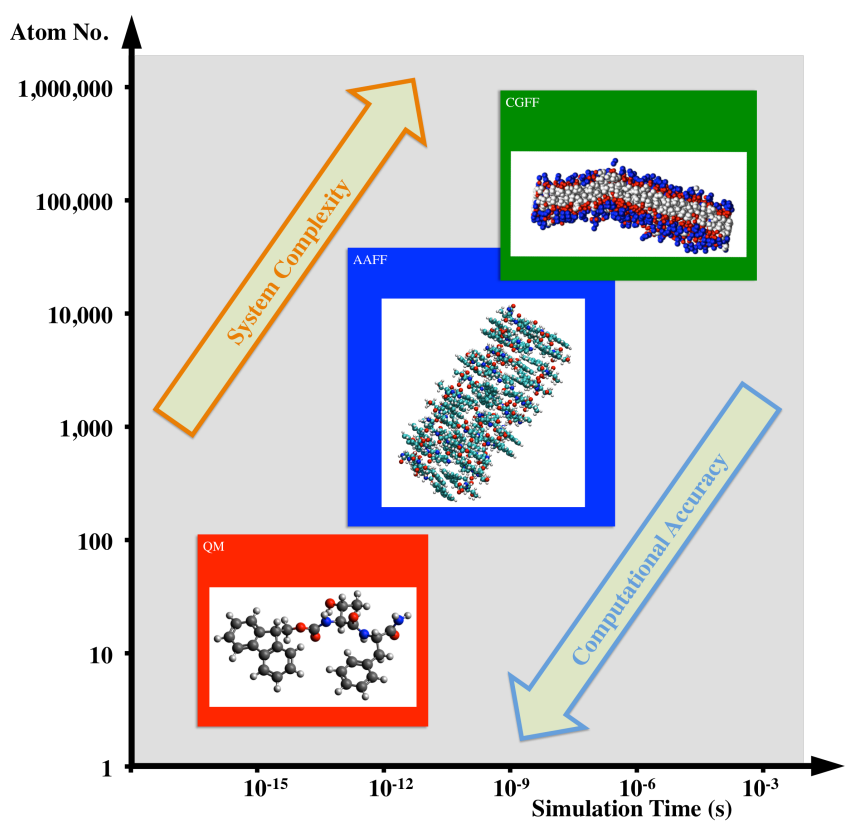

Figure 4. Comparison of the utility of computational methods across varying time and size regimes. $\mathrm{QM}=$ Quantum mechanics, AAFF = All-atom force-fields, CGFF = Coarse grained force-fields.

\section{Quantum Chemistry}

Quantum mechanical (QM) methods offer the promise of high accuracy and detailed, unbiased insights into the supramolecular structures that are formed from peptidebased building blocks. By determining the wavefunction and solving the Schrödinger equation for a given system all potentially observable information about the system can be calculated. Unfortunately, solving Schrödinger's equation for all possible nuclear arrangements to allow for a complete mapping of the potential energy surface for a nanostructure built from 100's and 1000's of peptide-based sub-units 
remains unfeasible, even with the impressive advances in algorithm efficiency and computing power that has been witnessed over the past twenty years. As such, the typical approach to studying peptide self-assembly with quantum mechanical methods has been to employ model systems.

The most widely used methodology in the QM study of peptides is density functional theory (DFT). While some studies do employ wavefunction based methodology, such as MP2, ${ }^{[15]}$ or make use of the simplicity of semi-empirical based methods such as OM3-D, ${ }^{[16]}$ the vast majority of studies utilise DFT. In most QM studies the object has been to understand the strength of specific interactions between monomers units, such as the H-bonding interactions or dispersion interactions, which in turn can be used to determine the most favoured orientation or stacking conformation of the monomer units. ${ }^{[15,17]}$ Owing to the cost of the QM calculations, these types of studies typically make use of dimer, tetramers and even up to octamers of the monomer sub-units to evaluate the binding energies and favoured structures and then extrapolate these finding to the larger nanostructure assuming a regular repeating unit. This type of structural and energetic information is particularly useful when considering how to modify a system or to design a new structure based on an existing model. An example case study from the literature that uses this approach is discussed below (Section 2.1).

\subsection{Preferred Packing Poses}

In their recent work on rigid cyclic $\gamma$-peptides, $\mathrm{Wu}$, Deng and co-workers utilized a combination of experimental and computational methods to determine the self-assembled structure of their peptide nanotubes. ${ }^{[17 \mathrm{~d}]}$ In this work the authors demonstrated that it was possible to synthesize the cyclic $\gamma$-tetrapeptide (1, Figure 5) through a cyclodimerization reaction of the parent dipeptide..$^{[17 \mathrm{~d}]}$
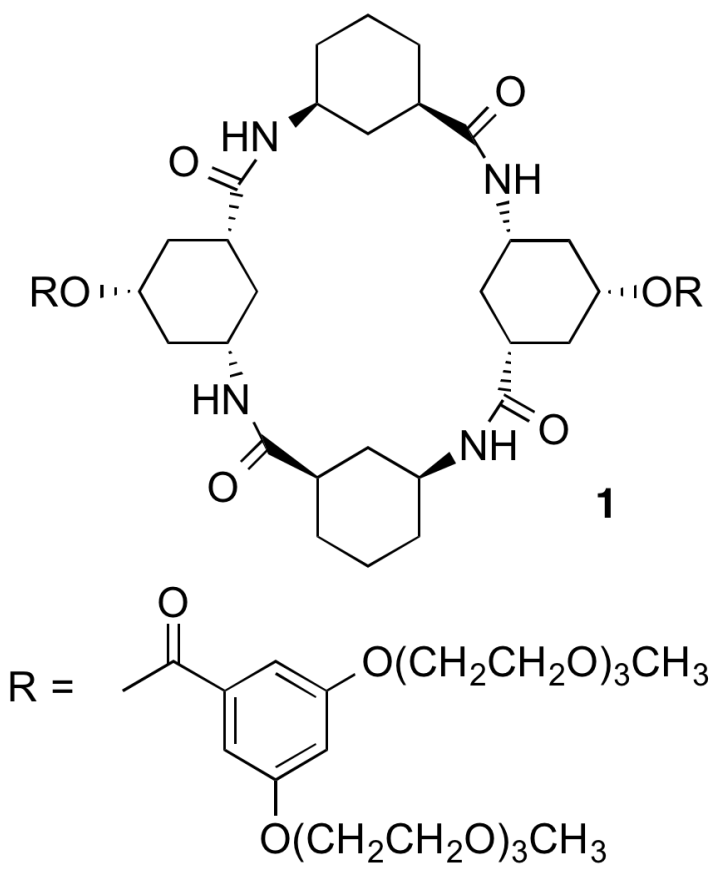

Figure 5. Molecular structure of 1.

The cyclic $\gamma$-tetrapeptide was found to self-assemble and form a colourless organogel when incubated in dichloromethane. After dispersing the organogel in water by sonication the authors were able to examine the systems by atomic force microscopy (AFM) and tunnelling electron microscopy (TEM). These two techniques were able to show that the peptides formed into long $(>1 \mu \mathrm{m})$ regular nanofibres with a height of $1.4-1.6 \mathrm{~nm}$ and a width of $\sim 5 \mathrm{~nm} .{ }^{[17 \mathrm{~d}]}$ This type of structural data from microscopy is able to provide important detail about the type of structures that can form and the dimensions of the fibres found in this case are consistent with a structure that have individually stacked units of $\mathbf{1}$. However, the interactions that allow this packing to occur cannot be resolved by these techniques. In order to determine the nature of the packing mechanism, the authors employed DFT studies to investigate the alternative motifs.

The optimisation of $\mathbf{1}$ revealed that there was only one dominant conformation of the cyclohexane rings, in the boat shape, and that the rigidity of the cyclic tetrapeptide meant that there was only one low-energy conformation for the backbone of the molecule. ${ }^{[17 \mathrm{~d}]}$ The structural rigidity of the building block is a clear benefit when designing the nanostructures that are able to form from these blocks. By limiting the number of potential conformers, the possible packing arrangements are also limited. In this case, the authors were only able to identify two possible ways that the rigid monomer would be able to interact with itself, either through parallel or anti-parallel $\beta$-sheet-like H-bonding patterns. ${ }^{[17 \mathrm{~d}]}$

In order to determine which of the two possible arrangements was preferred, the authors carried out optimisations of the two possible arrangements of dimers of 1 . The optimisations were carried out at the M05-2X/6-31+G(d,p) level of theory ${ }^{[18]}$ and the binding energies were corrected for basis set superposition error. In the gas phase, the parallel dimer was calculated as having a binding energy of $-34.4 \mathrm{kcal} / \mathrm{mol}$ and the antiparallel dimer with a binding energy of $-23.0 \mathrm{kcal} / \mathrm{mol}$. An analysis of the structures resulting from the optimisation revealed that the parallel dimer suffered less internal strain and was also able to form stronger $\mathrm{H}$ bonding interactions, relative to the anti-parallel dimer. ${ }^{[17 \mathrm{~d}]}$

This example case study shows the power of bringing together both experimental and computational methods to gain an understanding into the selfassembled structure of a peptide-based material. The authors were able to rely on a combination of experimental microscopy methods to determine the size of the system as well as FTIR data to provide information about the role of H-bonding interactions. However, the resulting two possible arrangements could not be distinguished by experimental methods alone. In this study, the distinction between the arrangements was instead carried out by considering their relative energetic stability. Given the rigidity of the cyclic $\gamma$ tetrapeptide this approach was tenable. However, in most cases, with non-cyclic building blocks, this is unlikely to be the case and therefore, evaluating potential arrangements through their relative binding energies can be problematic. Particularly if the energetic difference between several possible conformations is within the limits of the model applied. ${ }^{[19]}$ 
To overcome this problem, there has been an increasing shift towards the use of QM methods to predict properties that can be directly compared to the experimental observables. Experimentally, several techniques can be used to provide information about the types of non-covalent interactions that are involved in the self-assembled system. Using a variety of potential arrangements for the system, the equivalent spectra can be calculated and as such, the structure that correlates with the experimental spectra can be determined. Examples of this approach have been successfully employed for evaluating absorption spectra with TDDFT, ${ }^{[20]}$ calculating NMR chemical shifts to determine the conformation of a peptide, ${ }^{[16]}$ and calculating Fourier transform infrared (FTIR) spectra to determine the nature of the H-bonding interactions. ${ }^{[19,21]}$ An example case study of this approach is also discussed below (Section 2.2).

\subsection{Structures through Spectra}

In a combined experimental and computational approach, Fleming, et al., ${ }^{[19]}$ investigated the validity of assigning secondary structure motifs, parallel or anti-parallel $\beta$-sheet arrangements using FTIR. Within the field of protein structure determination, the amide I region of the IR spectra is known to be sensitive to changes in the H-bonding patterns of $\beta$-sheets. As such, the presence of $\beta$-sheets had been widely reported for a class of gelators composed from various short (di- and tri-) peptides capped at the N-terminus with an aromatic group; most commonly the Fmoc moiety. ${ }^{[13 c, 22]}$ In the case of proteins, it is possible differentiate between infinite parallel and antiparallel $\beta$ sheets, the former typically showing a single band at approximately $1615-1640 \mathrm{~cm}^{-1}$ and the latter having an additional component near $1685 \mathrm{~cm}^{-1}$. However, in the case of short peptides assembled into a nanostructure, the validity of the vibrational exciton running along a $\beta$-strand to produce the high frequency peak was unclear, given the short (2-3 amino acid) length of the strand. Therefore, in order to investigate the origin of the split amide I peaks in Fmoc-dipeptides a number of computational and experimental studies were performed. ${ }^{[19]}$

Initial calculations, at the B97-D/def2-SVP level of theory, ${ }^{[23]}$ of the vibrational modes of the Fmocdialanine (Fmoc-AA) monomer unit were carried out to determine the IR spectra of the individual building block. This allowed the determination of the spectra in the absence of $\mathrm{H}$-bonding that would be present in a self-assembled structure. From these calculations it was apparent that the split amide I peak was already present for the monomer and that the higher frequency peak was due to the presence of the carbamate moiety in Fmoc (Figure 6). In order to verify this, a second system was constructed which lacked the carbamate group, but was otherwise identical - Fluorenylmethylcarbonyldialanine (Fmc-AA, Figure 6). ${ }^{[19]}$

The presence of a split peak for the Fmoc-AA monomer could also be confirmed experimentally. By dissolving the compounds in methanol, where aggregation of the units is disfavoured, the IR spectra of the monomers could be obtained. Despite the fact that the calculated spectra clearly overestimated the frequency of the vibrational modes, due to model effects, the relative intensity and separation of the peaks in the amide I region were consistent between the computational and experimental data. ${ }^{[19]}$

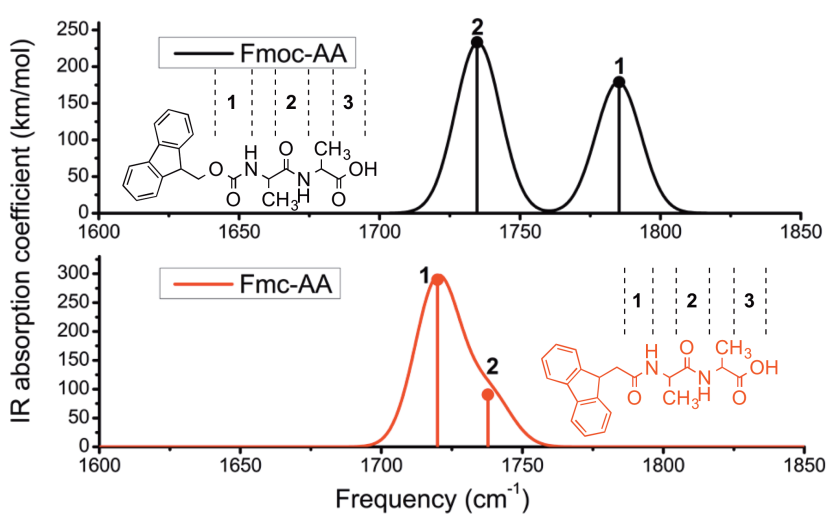

Figure 6. Simulated amide I spectra for Fmoc-AA and Fmc-AA monomers. Labels 1 and 2 indicate the number of the carbonyl giving the main contribution to the transition dipole moment Vibrations localised on carboxylic acid groups (carbonyl 3, generally $>1790 \mathrm{~cm}-1$ ) groups are omitted. ${ }^{[19]}$

Even though the splitting of the peaks in Fmoc-AA was not due to the presence of a vibrational exciton along the monomer, the shift of the primary amide I mode upon forming a nanostructure could still be diagnostic of the type of nanostructure being formed. Therefore, in addition to the study of the monomers, dimer and tetramer models of the Fmoc-AA and FmcAA in parallel and a variety of potential anti-parallel arrangements were constructed.

As well as vibrational calculations, binding energies were also computed for these model structures. $\beta$-sheets of Fmc-AA were found to be more stable than Fmoc-AA and the tetramers were more stable (on a per monomer basis) than the corresponding dimers due to cooperative binding effects. ${ }^{[19]}$ Furthermore, the authors showed that the parallel arrangement was generally found to be more stable than the antiparallel arrangement, although as stated previously the variations between the binding energies for flexible molecules such as these were small. Therefore, the differences in the relative binding energies of these arrangements were not considered to be good indicators for determining the equilibrium distribution of supramolecular structures. Rather, the correlation between the experimental and calculated vibrational spectra is a much more reliable indicator due to the local nature of the factors effecting the shifts in the amide I bands. ${ }^{[19]}$

Comparison of the calculated infrared bands for the different arrangements with the experimental spectra revealed several interesting features. When ignoring size-dependent artefacts in the DFT results, the lineshapes of the predicted absorption bands are in good agreement with experimental results. The experimental results consistently showed the amide I peak of Fmc-AA at a lower frequency than that seen for the infrared bands of Fmoc-AA. This observation suggested that the proximity of the fluorenyl group also influences the amide I vibration as was seen in the calculated spectra. ${ }^{[19]}$

Despite the fact that the calculated frequencies were systematically too high, it was apparent that, as the finite computational models become larger, from dimer 
to tetramers, a gradual convergence towards the experimental infrared bands was observed. ${ }^{[19]}$ It should be noted that the frequency results were not corrected for anharmonic effects and basis set truncation, which usually leads to an overestimation of calculated vibrational frequencies.

Finally, the authors were able to show that the antiparallel tetramer results more closely resembled the experimental infrared spectra when compared with the analogous parallel tetramer model, which suggested that the antiparallel arrangement is the most accurate depiction of the supramolecular structure. ${ }^{[19]}$

\section{Atomistic Molecular Mechanics}

The most common approach to modeling the self-assembly of peptide-based nanostructures is through the use of atomistic molecular mechanics. The use of all atom forcefields (AAFF) for studying biomolecular systems is wellestablished in the literature ${ }^{[24]}$ and as such the extension of these force-fields to study the processes of self-assembly in peptide-based systems is clearly justified. The use of AAFF provides the opportunity to study much larger systems, relative to the QM methods, and to include effects such as specific solvation which are considered critical to the selfassembly of this class of systems. However, despite the relative efficiency of the force-fields approach there remain significant time and size restrictions in modeling the selfassembly mechanism.

As mentioned above, the use of QM methodology to study self-assembly is typically limited to studying specific interactions in model systems of $1-12$ subunits, in the gas phase, or using an implicit solvent model. Whereas, in AAFF, it is routinely possible to model $50-150$ monomers in a fully solvated system. However, despite the 1-2 orders of magnitude increase in the system size, these models are still significantly smaller than the nanostructures observed experimentally in all but a few cases. As such, the AAFF models of nanostructures typically suffer from "edge-effects", that is, a much larger percentage of the modelled nanostructures is exposed to the solvent, relative to the real system. Moreover, the timescale of the AAFF molecular dynamics (MD) simulations is typically on the 10's - 100's of nanoseconds, which is much shorter than the experimental timescale for self-assembly. ${ }^{[25]}$ Therefore, in order to provide insights into the selfassembled structures a number of simplifying approximations are typically employed.

Given the short timescales that can be accessed using AAFF, the most common approach to studying the selfassembled nanostructures is to construct a proposed final structure of the system and then to test the stability of this structure through MD simulations. ${ }^{[26]}$ This approach is particularly useful in confirming the validity of proposed structures based on experimental data that also provides structural insight, such as IR, UV-Vis absorption, circular dichroism, etc. However, it is also possible for AAFF MD studies to generate potential structures by carrying out longer timescale (100 ns -1 $\mu \mathrm{s})$ simulations on smaller systems (typically 10's of monomers) to gain an insight into what the dominant interactions between the sub-units and the stability of the resulting aggregates. This approach has been particularly successful when considering the selfassembly of peptide-based systems on surfaces. ${ }^{[27]}$

\subsection{Structures through Simulation}

An excellent example of the types of information that can be acquired from AAFF simulations was published by Schatz, et al. in 2011 in their study of cylindrical nanofibres. ${ }^{[26 f]}$ In this work, the authors performed a MD simulation with the CHARMM force-field ${ }^{[24 \mathrm{f}]}$ of a cylindrical nanofibre composed of 144 peptide amphiphiles (PAs) with explicit solvent and counterions. The PA in this case consisted of a lipophilic hydrocarbon tail attached to the N-terminus of a 15-mer peptide containing charged amino acids (Figure 7). The PAs were arranged in a cylindrical pattern with nine PAs forming one layer with the overall nanostructure containing 16 layers. ${ }^{[26 \mathrm{f}]}$ The nanostructure was placed in a large $\left(144 \times 144 \times 84 \AA^{3}\right)$ box of $\sim 30,000$ water molecules, $162 \mathrm{Na}^{+}$ions and $18 \mathrm{Cl}^{-}$ions. The very large size of the system effectively restricted the amount of simulation time that could be achieved and after an initial equilibration period the system was simulated using the NPT ensemble for $40 \mathrm{~ns}$. However, the information available about the overall structure (i.e., that the PAs formed a cylindrical nanofibre) suggested that the relatively short simulation time would still produce meaningful information as the initial aggregation process was carried out manually in the system

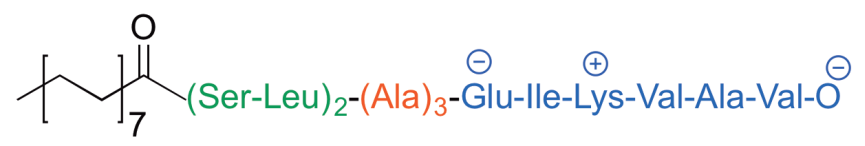
construction.

Figure 7. Structure of the peptide amphiphile (PA). The lipophilic segment is in black, the $\beta$-sheet segment is in green, the spacer segment is in red and the epitope segment is in blue.

The authors were able to demonstrate that, despite the short simulation length, the fibre was stable for an extended period of the simulation. The initial fibre radius for the starting structure was $\sim 48 \AA$, however, the system smoothly contracted over a $20 \mathrm{~ns}$ time period such that for the second period of the simulation the radius remained stable at $\sim 44 \AA,{ }^{[26 \mathrm{f}]}$ which was consistent with the available experimental data. ${ }^{[28]}$ Around the same time point $(\sim 20 \mathrm{~ns})$ in the simulation the non-bonded energy per PA also stabilised, after increasing by $\sim 20 \mathrm{kcal} / \mathrm{mol}$ from the starting system configuration, ${ }^{[26 f]}$ indicating that an equilibrium structure had been obtained.

In addition to structural insights, one of the powerful features of AAFF simulations is the capability of directly quantifying the relative strengths of interactions that stabilise the structures. In this case, the authors were able to determine that the electrostatic interactions between the PAs was actually destabilising - due to the net anionic charge of the PAs. However, this repulsive interaction was offset by both the attractive van der Waals interactions between the PAs and the significant electrostatic interaction energy between the PAs and the surrounding counterions. ${ }^{[26 \mathrm{f}]}$ This observation was also consistent with the experimental data, which showed that the formation of the cylindrical aggregates was triggered by counterion screening. ${ }^{[29]}$ 
The agreement with experiment that could be achieved by analysing the energetic and structural data is impressive. However, for computational approaches to be truly useful they need to offer unique insights that are not available from alternative experimental approaches. In this respect one of the key findings of this work is the amount of disorder that an apparently well-ordered system is able to tolerate. The classical view of nanostructured systems is one of highly-order sub-units that have a regularly repeating stabilising interaction such as $\pi$-stacking and $\mathrm{H}$-bonding, in parallel or anti-parallel $\beta$-sheet arrangements. In contrast to this ordered, relatively static, view of the nanostructure, Schatz, et al., demonstrated that the nanofibre was able to maintain its cylindrical shape and dimensions with only $14 \% \beta$-sheet content (Table 1 ), predominantly with a parallel alignment. ${ }^{[26 \mathrm{f}]}$ This view that the relatively disordered, dynamic alignment of sub-units is able to lead to a well-defined nanostructure can have significant implications for the design of self-assembling building blocks.

Table 1. Average population of the secondary structure of PAs in the cylindrical fibre. ${ }^{[26 f]}$

\begin{tabular}{cc}
\hline Configuration & $\%$ \\
\hline$\alpha$-helix & 1 \\
$\beta$-sheet & 14 \\
Turn & 20 \\
Coil & 65 \\
\hline
\end{tabular}

\section{Coarse Grained Molecular Mechanics}

As the example above demonstrates, the AAFF approach is able to provide detailed structural and energetic data for nanostructured systems. However, in order to progress beyond analyzing systems with a known nanostructure requires knowledge of the self-assembly mechanism. This is the main limitation of the AAFF approach. The selfassembly of typical peptide-based systems occur over the $\mu \mathrm{s}$ - ms time scale and the resulting nanostructures can have widths of $5-100 \mathrm{~nm}$ and lengths on the $\mu \mathrm{m}$ scale. These time and size regimes are currently beyond the limits of our most advanced supercomputers and sophisticated algorithms, particularly for routine calculations. Therefore, in order to progress beyond the analysis of self-assembled structures and into the study of the process of self-assembly requires a different approach.

An emerging approach for tackling the large time and size regimes required in the study of peptide selfassembly is through the use of a coarse-grained forcefield (CGFF). ${ }^{[2,30]}$ Within this context, a CGFF typically implies that a number of atoms in the molecule are collectively represented by a single "bead". One of the most popular CGFFs for studying peptide-based selfassembly is the Martini force-field. ${ }^{[1]}$ The Martini force-field utilises a mapping of $\sim 4$ heavy atoms to each bead $(4: 1)$. For example, the non-hydrogen atoms that constitute the backbone atoms of an amino acid (-N-C$\mathrm{C}(=\mathrm{O})-\mathrm{C}-)$ are grouped into a single bead to represent the backbone (Figure 8), while the sidechain of phenylalanine is represented by a 2-3:1 mapping in order to maintain the flat structure of the aromatic group (Figure 8). The act of describing several atoms as a single bead has several beneficial factors, namely: (a) the reduction in the degrees of freedom in the system; (b) the reduction in the number of interacting particles; (c) a simplification of the potential energy expression; and (d) the larger mass of the beads, relative to the atoms, allows for much longer timestep ( $\sim 25 \mathrm{fs})$ relative to the atomistic simulations (1-2 fs). These factors combine to allow an increase in efficiency of two to three orders of magnitude over the AAFF simulations. Practically, this translates into the ability to routinely calculate explicitly solvated systems with 1000's of self-assembling monomers on the $\mu$ s timescale.
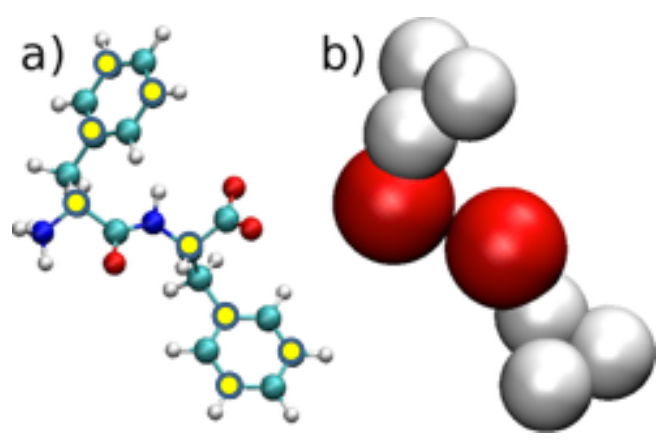

Figure 8. (a) All atom representation of diphenylalanine. The yellow circles indicate where the coarse-grain beads are positioned after mapping. (b) The coarse-grained representation of diphenylalanine. The red beads represent the backbone atoms and the white beads represent the sidechain atoms.

Clearly, the coarse-graining of a system results in a loss of information. The ability to represent specific interactions, such as H-bonding, is not possible with the loss of atomic detail. Therefore, it was of interest to determine whether such coarse grained approaches could still provide meaningful information about both the self-assembly mechanism of peptide-based systems and the resulting nanostructures.

\subsection{Mechanisms from Movies}

In 2011, Frederix, et al., investigated the ability of a CGFF to predict whether a given dipeptide would self-assemble or not. ${ }^{[2]}$ As described above, the simplifications inherent in a CGFF result in a significant increase in the efficiency of MD simulations and as such it was possible, for the first time, to effectively screen all 400 of the 20 gene-encoded dipeptides for their propensity to self-assemble. The initial screening involved the simulation of each dipeptide (in the zwitterionic form) using the Martini CGFF. Each simulation started from a cubic box of 300 randomly placed dipeptides, solvated in Martini coarse-grained water to a final concentration of $\sim 0.4 \mathrm{M}$. The simulations were carried out in the NPT ensemble with a 25 fs timestep for a simulation time of 100 ns. ${ }^{[2]}$ However, because of the smoothness of the $\mathrm{CG}$ potential this equates to an effective simulation time of $\sim 400$ ns. $^{[32]}$ The output of the 400 simulations were then analysed for their aggregation propensity (AP, defined as the ratio between the solvent accessible surface area of the peptides in the starting and final states of the simulation $)^{[2]}$ 
to determine whether the dipeptide was likely to selfassemble.

The AP scores resulting from these simulations revealed that the hydrophobic amino acids, phenylalanine and tryptophan, promote aggregation, whereas small or charged residues decrease the propensity. However, most dipeptides have an AP score close to 1, indicating that they do not exhibit a propensity to aggregate and as such are unlikely to form self-assembled nanostructures. The AP scores of the peptides were compared to literature examples of dipeptides and the simulation data was able to accurately predict the ability of the known dipeptides to aggregate, or not.

The ability of the CGFF methods to predict whether a peptide will aggregate clearly has many potential applications. However, this screening protocol, which utilises relatively short time and size scales for CGFFs, was not extensive enough to indicate the ability of the Martini CGFF to describe the self-assembly mechanism and nor the resulting nanostructure. Therefore, for the FF dipeptide, which has been well characterized experimentally, a longer, larger simulation was performed.

The extended simulation of FF started from a cubic box of 1600 randomly placed FF molecules, solvated in Martini coarse-grained water to a final concentration of $\sim 0.4$ M. Under the same conditions used in the screening simulations, the MD simulation was carried out for $1.5 \mu \mathrm{s}$.

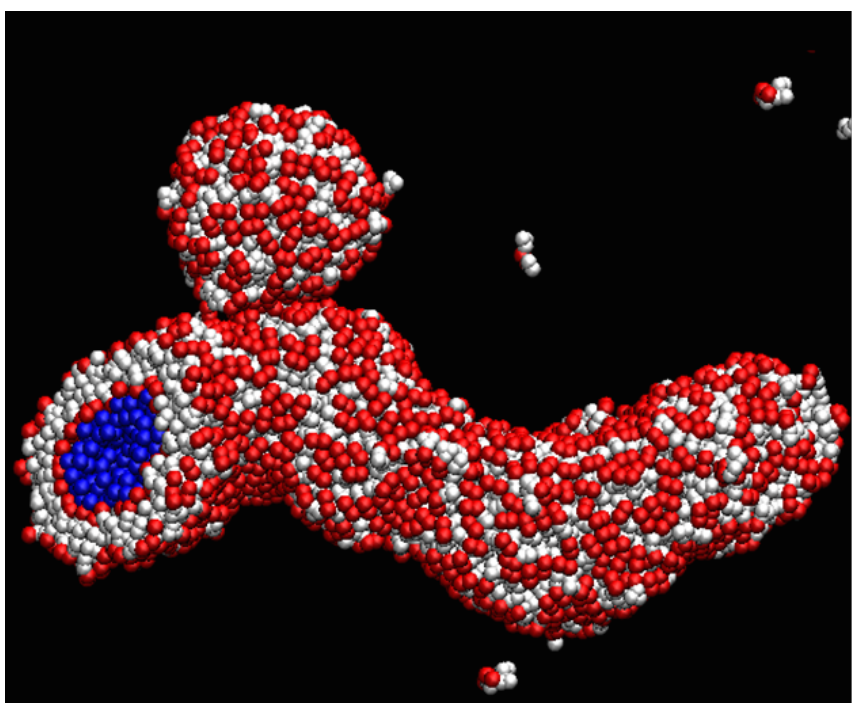

Figure 9. Final snapshot of the $1.5 \mu \mathrm{s}$ simulation of FF. Red beads represent the backbone, white beads represent the sidechain. The end of the tube is cut off to show water beads (blue) on the interior. Water beads outside of the tube are omitted for clarity.

In the final snapshot of the $1.5 \mu$ s simulation the FF monomers generated a tubular nanostructure (Figure 9) in agreement with that observed experimentally. The dimensions of the 1D nanotube (outer and inner diameters) also agreed reasonably well with the X-ray diffraction analysis of crystallized FF nanotubes. ${ }^{[5]}$ In addition to the overall dimensions of the nanotubes it was also possible to correlate the rotation of the sidechains of the dipeptides with the experimentally known structure that is adopted. The dihedral angle between the side chains was observed to rotate from $180^{\circ}$ in the starting monomer structures to an average value of $0^{\circ}$ in the final tube like nanostructure.

In addition to the final tubular structure, the simulation also provided new information with respect to the mechanism by which the nanotubes are formed. From the initial random distribution, the dipeptides were observed to form sheet-like aggregates after $0.2 \mu \mathrm{s}$. The aggregate structures continued to accumulate more monomers up to the point where they were able to fold up to form hollow vesicles $(\sim 0.5 \mu \mathrm{s})$. The hollow vesicles that were formed continued to merge for the duration of the simulation, resulting in the extended, 1D nanotube after $1.5 \mu \mathrm{s}$.

\section{Summary}

The self-assembly of peptide-based systems is rapidly emerging as technique with enormous potential applications to a variety of technological challenges. The minimalistic design of new nanostructures based on these biocompatible sub-units has already made significant progress through the experimental discovery of a number of interesting systems. However, for this rate of discovery to be maintained and hopefully accelerated the serendipitous discovery and empirical design of systems needs to be replaced by a rational understanding and a design process based on this understanding. The use of computational models can aid in this transition by: providing insights into the atomic level details of the self-assembled structures; determining the molecular forces that drive the self-assembly and stabilize the resulting structures; and insights into not only the structure but also the assembly process itself.

\section{References}

[1] S. Fleming, R. V. Ulijn, Chem. Soc. Rev. 2014, 43, 81508177.

[2] P. W. J. M. Frederix, R. V. Ulijn, N. T. Hunt, T. Tuttle, J. Phys. Chem. Lett. 2011, 2, 2380-2384.

[3] S. Zhang, T. Holmes, C. Lockshin, A. Rich, Proc. Natl. Acad. Sci. U. S. A. 1993, 90, 3334-3338.

[4] a) M. Reches, E. Gazit, Science 2003, 300, 625-627; b) C. H. Gorbitz, Chem. Eur. J 2001, 7.

[5] C. H. Gorbitz, Chem. Commun. 2006, 2332-2334.

[6] a) M. Reches, E. Gazit, Nano Lett. 2004, 4; b) S. Scanlon, A. Aggeli, Nano Today 2008, 3, 22-30.

[7] a) G. Colombo, P. Soto, E. Gazit, Trends in biotechnology 2007, 25, 211-218; b) S.-J. Rymer, A. T. B. Tendler, C. Bosquillon, C. Washington, C. J. Roberts, Ther. Deliv. 2011, 2, 1043-1056.

[8] P. Tamamis, L. Adler-Abramovich, M. Reches, K. Marshall, P. Sikorski, L. Serpell, E. Gazit, G. Archontis, Biophys. J 2009, 96, 5020-5029.

[9] T. H. Han, T. Ok, J. Kim, D. O. Shin, H. Ihee, H. S. Lee, S. O. Kim, Small 2010, 6, 945-951.

[10] a) S. Marchesan, C. D. Easton, F. Kushkaki, L. Waddington, P. G. Hartley, Chem. Commun. 2012, 48, 2195-2197; b) S. Marchesan, C. D. Easton, K. E. Styan, L. J. Waddington, F. Kushkaki, L. Goodall, K. M. McLean, J. S. Forsythe, P. G. Hartley, Nanoscale 2014, 6, 5172-5180; c) S. Marchesan, Y. Qu, L. J. Waddington, C. D. Easton, V. Glattauer, T. J. Lithgow, K. M. McLean, J. S. Forsythe, P. G. Hartley, Biomaterials 2013, 34, 3678-3687; d) S. Marchesan, L. Waddington, C. D. Easton, F. Kushkaki, K. M. McLean, J. S. Forsythe, P. G. Hartley, BioNanoScience 2013, 3, 21-29; e) S. Marchesan, L. Waddington, C. D. 
Easton, D. A. Winkler, L. Goodall, J. Forsythe, P. G. Hartley, Nanoscale 2012, 4, 6752-6760; f) W.-W. Tsai, L.-S. Li, H. Cui, H. Jiang, S. I. Stupp, Tetrahedron 2008, 64, 8504-8514.

[11] M. Cao, C. Cao, L. Zhang, D. Xia, H. Xu, J. Colloid Interface Sci. 2013, 407, 287-295.

[12] C. Tanford, The Hydrophobic Effect: Formation of Micelles and Biological Membranes $2 d$ Ed, J. Wiley., 1980 .

[13] a) J. B. Blanco-Canosa, P. E. Dawson, Angew. Chem. Int. Ed. 2008, 47, 6851-6855; b) S. Debnath, A. Shome, D. Das, P. K. Das, J. Phys. Chem. B 2010, 114, 4407-4415; c) V. Jayawarna, S. M. Richardson, A. R. Hirst, N. W. Hodson, A. Saiani, J. E. Gough, R. V. Ulijn, Acta Biomat. 2009, 5, 934-943; d) Y. Shin, K. A. Winans, B. J. Backes, S. B. H. Kent, J. A. Ellman, C. R. Bertozzi, J. Am. Chem. Soc. 1999, 121, 11684-11689; e) Y. Zou, K. Razmkhah, N. P. Chmel, I. W. Hamley, A. Rodger, RSC Adv. 2013, 3, 10854-10858.

[14] a) V. Castelletto, G. Cheng, B. W. Greenland, I. W. Hamley, Langmuir 2011, 27, 2980-2988; b) H. Zhang, H. Wang, G. Xu, S. Yuan, Colloids Surf., A 2013, 417, 217 223.

[15] C. Liu, D. X. Zhao, Z. Z. Yang, J. Comput. Chem. 2012, 33, 379-390.

[16] T. Tuttle, J. Phys. Chem. A 2009, 113, 11723-11733.

$[17]$ a) T. Beke, A. Czajlik, B. Balint, A. Perczel, ACS Nano 2008, 2, 545-553; b) Y. F. Chen, J. J. Dannenberg, $J$. Comput. Chem. 2011, 32, 2890-2895; c) I. A. W. Filot, A. R. A. Palmans, P. A. J. Hilbers, R. A. van Santen, E. A. Pidko, T. F. A. de Greef, J. Phys. Chem. B 2010, 114, 13667-13674; d) L. C. Li, H. M. Zhan, P. F. Duan, J. Liao, J. M. Quan, Y. Hu, Z. Z. Chen, J. Zhu, M. H. Liu, Y. D. Wu, J. G. Deng, Adv. Funct. Mater. 2012, 22, 3051-3056; e) W. W. Qu, H. W. Tan, G. J. Chen, R. Z. Liu, Int. J. Quantum Chem. 2010, 110, 1648-1659; f) M. Rauschenberg, S. Bandaru, M. P. Waller, B. J. Ravoo, Chem. Eur. J. 2014, 20, 2770-2782; g) Z. F. Sun, Z. Y. Li, Y. H. He, R. J. Shen, L. Deng, M. H. Yang, Y. Z. Liang, Y. Zhang, J. Am. Chem. Soc. 2013, 135, 13379-13386.

[18] a) R. Garcia-Fandino, L. Castedo, J. R. Granja, S. A. Vazquez, J. Phys. Chem. B 2010, 114, 4973-4983; b) Y. Zhao, N. E. Schultz, D. G. Truhlar, J. Chem. Theory Comput. 2006, 2, 364-382; c) Y. Zhao, D. G. Truhlar, J. Chem. Theory Comput. 2007, 3, 289-300.

[19] S. Fleming, P. W. J. M. Frederix, I. R. Sasselli, N. T. Hunt, R. V. Ulijn, T. Tuttle, Langmuir 2013, 29, 95109515 .

[20] S. K. Murase, N. Haspel, L. J. del Valle, E. A. Perpete, C. Michaux, R. Nussinov, J. Puiggali, C. Aleman, RSC Adv. 2014, 4, 23231-23241.

[21] W. Nuansing, A. Rebollo, J. M. Mercero, J. Zuniga, A. M. Bittner, J. Raman Spectrosc. 2012, 43, 1397-1406.

[22] a) V. Castelletto, G. Cheng, B. W. Greenland, I. W. Hamley, P. J. F. Harris, Langmuir 2011, 27, 2980-2988; b) S. Debnath, A. Shome, D. Das, P. K. Das, J. Phys. Chem. $B$ 2010, 114, 4407-4415; c) A. R. Hirst, S. Roy, M. Arora, A. K. Das, N. Hodson, P. Murray, S. Marshall, N. Javid, J. Sefcik, J. Boekhoven, J. H. van Esch, S. Santabarbara, N. T. Hunt, R. V. Ulijn, Nat. Chem. 2010, 2, 1089-1094; d) R. Orbach, I. Mironi-Harpaz, L. Adler-Abramovich, E. Mossou, E. P. Mitchell, V. T. Forsyth, E. Gazit, D. Seliktar, Langmuir 2012, 28, 2015-2022; e) A. M. Smith, R. J. Williams, C. Tang, P. Coppo, R. F. Collins, M. L. Turner, A. Saiani, R. V. Ulijn, Adv. Mater. 2008, 20, 37+; f) R. J. Williams, A. M. Smith, R. Collins, N. Hodson, A. K. Das, R. V. Ulijn, Nat. Nanotechnol. 2009, 4, 19-24; g) H. X. Xu, A. K. Das, M. Horie, M. S. Shaik, A. M. Smith, Y. Luo, X. F. Lu, R. Collins, S. Y. Liem, A. M. Song, P. L. A. Popelier, M. L. Turner, P. Xiao, I. A. Kinloch, R. V. Ulijn, Nanoscale 2010, 2, 960-966; h) X. D. $\mathrm{Xu}$, C. S. Chen, B. Lu, S. X. Cheng, X. Z. Zhang, R. X. Zhuo, J. Phys. Chem. B 2010, 114, 2365-2372.

[23] a) S. Grimme, J. Comput. Chem. 2006, 27, 1787-1799; b) A. Schafer, H. Horn, R. Ahlrichs, J. Chem. Phys. 1992, 97, 2571-2577.
[24] a) B. R. Brooks, C. L. Brooks, A. D. Mackerell, L. Nilsson, R. J. Petrella, B. Roux, Y. Won, G. Archontis, C. Bartels, S. Boresch, A. Caflisch, L. Caves, Q. Cui, A. R. Dinner, M. Feig, S. Fischer, J. Gao, M. Hodoscek, W. Im, K. Kuczera, T. Lazaridis, J. Ma, V. Ovchinnikov, E. Paci, R. W. Pastor, C. B. Post, J. Z. Pu, M. Schaefer, B. Tidor, R. M. Venable, H. L. Woodcock, X. Wu, W. Yang, D. M. York, M. Karplus, J. Comput. Chem. 2009, 30, 1545-1614; b) D. A. Case, T. E. Cheatham, T. Darden, H. Gohlke, R. Luo, K. M. Merz, A. Onufriev, C. Simmerling, B. Wang, R. J. Woods, J. Comput. Chem. 2005, 26, 1668-1688; c) W. D. Cornell, P. Cieplak, C. I. Bayly, I. R. Gould, K. M. Merz, D. M. Ferguson, D. C. Spellmeyer, T. Fox, J. W. Caldwell, P. A. Kollman, J. Am. Chem. Soc. 1995, 117, 5179-5197; d) W. L. Jorgensen, D. S. Maxwell, J. TiradoRives, J. Am. Chem. Soc. 1996, 118, 11225-11236; e) A. D. Mackerell, J. Comput. Chem. 2004, 25, 15841604; f) A. D. MacKerell, D. Bashford, M. Bellott, R. L. Dunbrack, J. D. Evanseck, M. J. Field, S. Fischer, J. Gao, H. Guo, S. Ha, D. Joseph-McCarthy, L. Kuchnir, K. Kuczera, F. T. K. Lau, C. Mattos, S. Michnick, T. Ngo, D. T. Nguyen, B. Prodhom, W. E. Reiher, B. Roux, M. Schlenkrich, J. C. Smith, R. Stote, J. Straub, M. Watanabe, J. Wiorkiewicz-Kuczera, D. Yin, M. Karplus, J. Phys. Chem. B 1998, 102, 3586-3616; g) S. L. Mayo, B. D. Olafson, W. A. Goddard, J. Phys. Chem. 1990, 94, 88978909 ; h) J. W. Ponder, D. A. Case, Protein Simulations 2003, 66, 27-+; i) D. Van der Spoel, E. Lindahl, B. Hess, G. Groenhof, A. E. Mark, H. J. C. Berendsen, J. Comput. Chem. 2005, 26, 1701-1718; j) J. M. Wang, R. M. Wolf, J. W. Caldwell, P. A. Kollman, D. A. Case, J. Comput. Chem. 2004, 25, 1157-1174; k) S. J. Weiner, P. A. Kollman, D. A. Case, U. C. Singh, C. Ghio, G. Alagona, S. Profeta, P. Weiner, J. Am. Chem. Soc. 1984, 106, 765-784; 1) S. J. Weiner, P. A. Kollman, D. T. Nguyen, D. A. Case, J. Comput. Chem. 1986, 7, 230-252.

[25] G. Colombo, P. Soto, E. Gazit, Trends Biotechnol. 2007, 25, 211-218.

[26] a) K. M. Eckes, X. J. Mu, M. A. Ruehle, P. Y. Ren, L. J. Suggs, Langmuir 2014, 30, 5287-5296; b) M. Engels, D. Bashford, M. R. Ghadiri, J. Am. Chem. Soc. 1995, 117, 9151-9158; c) M. Hughes, P. Frederix, J. Raeburn, L. S. Birchall, J. Sadownik, F. C. Coomer, I. H. Lin, E. J. Cussen, N. T. Hunt, T. Tuttle, S. J. Webb, D. J. Adams, R. V. Ulijn, Soft Matter 2012, 8, 5595-5602; d) M. Hughes, H. X. Xu, P. Frederix, A. M. Smith, N. T. Hunt, T. Tuttle, I. A. Kinloch, R. V. Ulijn, Soft Matter 2011, 7, 1003210038; e) O. S. Lee, Y. M. Liu, G. C. Schatz, J. Nanopart. Res. 2012, 14; f) O. S. Lee, S. I. Stupp, G. C. Schatz, J. Am. Chem. Soc. 2011, 133, 3677-3683; g) J. Montenegro, M. R. Ghadiri, J. R. Granja, Acc. Chem. Res. 2013, 46, 2955-2965; h) K. L. Morris, A. Rodger, M. R. Hicks, M. Debulpaep, J. Schymkowitz, F. Rousseau, L. C. Serpell, Biochem. J. 2013, 450, 275-283; i) X. J. Mu, K. M. Eckes, M. M. Nguyen, L. J. Suggs, P. Y. Ren, Biomacromolecules 2012, 13, 3562-3571; j) Y. Mu, M. Yu, Soft Matter 2014, 10, 4956-4965; k) S. Tsonchev, A. Troisi, G. C. Schatz, M. A. Ratner, Nano Lett. 2004, 4, 427-431; 1) S. Tsonchev, A. Troisi, G. C. Schatz, M. A. Ratner, J. Phys. Chem. B 2004, 108, 15278-15284; m) D. Van Gough, J. S. Wheeler, S. F. Cheng, M. J. Stevens, E. D. Spoerke, Langmuir 2014, 30, 9201-9209; n) R. Vijayaraj, S. Van Damme, P. Bultinck, V. Subramanian, Phys. Chem. Chem. Phys. 2012, 14, 15135-15144; o) T. Yu, O. S. Lee, G. C. Schatz, J. Phys. Chem. A 2013, 117, 7453-7460; p) Y. R. Zhao, J. Q. Wang, L. Deng, P. Zhou, S. J. Wang, Y. T. Wang, H. Xu, J. R. Lu, Langmuir 2013, 29, 13457-13464; q) Y. Raz, B. Rubinov, M. Matmor, H. Rapaport, G. Ashkenasy, Y. Miller, Chem. Commun. (Cambridge, U. K.) 2013, 49, 6561-6563.

[27] a) C. R. L. Chapman, E. C. M. Ting, A. Kereszti, I. Paci, J. Phys. Chem. C 2013, 117, 19426-19435; b) R. Di Felice, S. Corni, J. Phys. Chem. Lett. 2011, 2, 1510-1519; c) H. Heinz, J. Comput. Chem. 2010, 31, 1564-1568; d) H. Heinz, B. L. Farmer, R. B. Pandey, J. M. Slocik, S. S. Patnaik, R. Pachter, R. R. Naik, J. Am. Chem. Soc. 2009, 
131, 9704-9714; e) H. Heinz, K. C. Jha, J. LuettmerStrathmann, B. L. Farmer, R. R. Naik, J. Roy. Soc. Interface 2011, 8, 220-232; f) B. Kim, S. J. Choi, S. H. Han, K. Y. Choi, Y. B. Lim, Chem. Commun. (Cambridge, U. K.) 2013, 49, 7617-7619; g) M. S. Liberato, S. Kogikoski, E. R. Silva, M. D. Coutinho-Neto, L. P. B. Scott, R. H. Silva, V. X. Oliveira, R. A. Ando, W. A. Alves, J. Phys. Chem. B 2013, 117, 733-740; h) A. K. Nowinski, F. Sun, A. D. White, A. J. Keefe, S. Y. Jiang, J. Am. Chem. Soc. 2012, 134, 6000-6005; i) C. R. So, J. L. Kulp, E. E. Oren, H. Zareie, C. Tamerler, J. S. Evans, M. Sarikaya, ACS Nano 2009, 3, 1525-1531.

[28] E. T. Pashuck, H. G. Cui, S. I. Stupp, J. Am. Chem. Soc. 2010, 132, 6041-6046.

[29] J. C. Stendahl, M. S. Rao, M. O. Guler, S. I. Stupp, Adv. Funct. Mater. 2006, 16, 499-508.

[30] a) B. Barz, B. Urbanc, J. Phys. Chem. B 2014, 118, 3761-3770; b) G. Bellesia, J. E. Shea, J. Chem. Phys. 2007, 126 ; c) S. P. Carmichael, M. S. Shell, J. Phys. Chem. B 2012, 116, 8383-8393; d) Y. Cote, I. W. Fu, E. T. Dobson, J. E. Goldberger, H. D. Nguyen, J. K. Shen, J. Phys. Chem. C 2014, 118, 16272-16278; e) O. Engin, A. Villa, C. Peter, M. Sayar, Macromol. Theory Simul. 2011, 20, 451-465; f) C. Guo, Y. Luo, R. H. Zhou, G. H. Wei, ACS Nano 2012 6, 3907-3918; g) C. Guo, Y. Luo, R. H. Zhou, G. H. Wei, Nanoscale 2014, 6, 2800-2811; h) S. Jun, Y. Hong, H. Received: ((will be filled in by the editorial staff)) Accepted: ((will be filled in by the editorial staff)) Published online: ((will be filled in by the editorial staff))
Imamura, B. Y. Ha, J. Bechhoefer, P. Chen, Biophys. J. 2004, 87, 1249-1259; i) E. Khurana, R. H. DeVane, A. Kohlmeyer, M. L. Klein, Nano Lett. 2008, 8, 3626-3630; j) O. S. Lee, V. Cho, G. C. Schatz, Nano Lett. 2012, 12, 4907-4913; k) D. Matthes, V. Gapsys, B. L. de Groot, $J$. Mol. Biol. 2012, 421, 390-416; 1) J. Mondal, B. J. Sung, A. Yethiraj, J. Chem. Phys. 2010, 132; m) M. Seo, S. Rauscher, R. Pomes, D. P. Tieleman, J. Chem. Theory Comput. 2012, 8, 1774-1785; n) Y. S. Velichko, S. I. Stupp, M. O. de la Cruz, J. Phys. Chem. B 2008, 112, 2326-2334; o) A. Villa, C. Peter, N. F. A. van der Vegt, Phys. Chem. Chem. Phys. 2009, 11, 2077-2086; p) A. Villa, N. F. A. van der Vegt, C. Peter, Phys. Chem. Chem. Phys. 2009, 11, 2068-2076; q) T. Yu, G. C. Schatz, J. Phys. Chem. B 2013, 117, 14059-14064; r) P. W. J. M. Frederix, G. G. Scott, Y. M. Abul-Haija, D. Kalafatovic, C. G. Pappas, N. Javid, N. T. Hunt, R. V. Ulijn, T. Tuttle, Nat. Chem. 2015, 7, 30-37.

[31] L. Monticelli, S. K. Kandasamy, X. Periole, R. G. Larson, D. P. Tieleman, S. J. Marrink, J. Chem. Theory Comput. 2008, 4, 7830-7843.

[32] S. J. Marrink, H. J. Risselada, S. Yefimov, D. P. Tieleman, A. H. De Vries, J. Phys. Chem. B 2007, 111, 13. 
